Conclusion: Further studies are needed to explore how varied factors interplay in the decision-making process of older adults in EOL care.

\section{MEASURING STAFF PERCEPTION OF END-OF-LIFE EXPERIENCE OF OLDER ADULTS IN LONG-TERM CARE}

N. Cornally ${ }^{1}$, A. Coffey ${ }^{1}$, D.W. Molloy ${ }^{1}$, R. O'Caoimh ${ }^{1,2}$, R. O'Sullivan ${ }^{1}$, C. McGlade ${ }^{1}$, 1. University College Cork, Cork, Ireland, Ireland, 2. NUI Galway, Galway, Ireland

Background: Quality of dying and death receive far less attention than quality of life. Measuring the quality of care at end-of-life (EOL) in long-term care (LTC) is essential, to ensure high standards.

Methods: A questionnaire measuring Staff Perception of their patient's End of Life Experience (SPELE) was developed. Content Validity (CVI) was assessed by a panel of experts and piloting was conducted with dyads of healthcare assistants $(\mathrm{n}=15)$ and nurses $(\mathrm{n}=15)$.

Results: The SPELE captures facets of the quality of the death and dying experience from healthcare staff's perspective. Good group inter-rater reliability was observed among subscales. One exception was the pain and symptom experience scale. Kappa values showed little agreement between nurses and healthcare assistants for certain symptoms, including pain.

Conclusion: Further testing of the questionnaire is underway in a large multi-centre randomised control trial in Ireland. To date the tool is described as a useful mechanism to enable researchers and clinicians to explore quality of care at EOL.

\section{SESSION 855 (POSTER)}

\section{FALLS I}

\section{SLEEP APNEA, FALLS AND SARCOPENIA IN OLDER ADULTS: PRELIMINARY RESULTS FROM FALL- AGING- SLEEP STUDY}

A. Monti ${ }^{1}$, M. Doulazmi, A. Breining4, V. Nguyen-Michel ${ }^{1}$, E. Pautas ${ }^{1}$, J. Mariani ${ }^{1}$, K. Kinugawa ${ }^{1}, 1$. AP-HP, DHU FAST, GH Pitie-Salpêtrière-Charles Foix, Paris, France, 2. Sorbonne Universités, UPMC University Paris 6, Paris, France, 3. CNRS, UMR 8256 Biological Adaptation and Aging, Paris, France, 4. Assistance Publique des Hôpitaux de Paris, Acute geriatric care, Pitié-Salpétrière-Charles-Foix Hospita, Ivry Sur Seine, France, 5. Assistance Publique des Hôpitaux de Paris, Functional explorations laboratory, Pitié-Salpétrière-Charles-Foix Hospital, Ivry Sur Seine, France

Objectives: Sleep disturbances increase the risk of falls among older people. We aimed to examine prevalence of falls and sarcopenia among older patients with and without sleep apnea (SA).

Methods: Acute care setting patients aged $\geq 75$ were proposed to participate to the FALL-A-SLEEP Study since March 2015. Subjective sleep questionnaires (e.g Epworth Sleepiness Scale (ESS)), nocturnal polygraphy (SA defined by $\mathrm{AHI}>15 / \mathrm{hr}$ ), handgrip strength and short physical performance battery (SPPB), Dual Energy X-ray absorptiometry (skeletal muscle mass (SMI)), were performed in a stabilized medical situation.

Results: Complete evaluation was available for 45 patients (mean age 81.9 years, 33 women). Between SA ( $\mathrm{n}=28$, mean $\mathrm{AHI}=39.7 / \mathrm{hr})$ and non-SA $(\mathrm{n}=17$, mean $\mathrm{AHI}=4.6 / \mathrm{hr})$ patients, nap was more frequent among SA patients $(65.51 \%$ vs $29.41 \%$, p-value $=0.023$ ) but ESS ( 5.9 vs 4.9 , $\mathrm{p}$-value $=0.275$ ) was not different. ADL (5.56 vs 5.71, p=0.883), Charlson score ( 1.7 vs $2.47, \mathrm{p}=0.301$ ), and Rockwood score (4.37 vs $4.29, \mathrm{p}=0.861)$ were not different. Falls $(77.7 \%$ vs $56.25 \%$, $\mathrm{p}$-value $=0.137)$, mean SPPB score $(5.3 / 12$ vs $7.3 / 12$, $\mathrm{p}$-value $=0.0771), S M I(7.03 \mathrm{~kg} / \mathrm{m} 2$ vs $6.17, \mathrm{p}$-value $=0.603)$, mean handgrip $(17.83 \mathrm{~kg}$ vs 17.97 , p-value $=0.799)$ and sarcopenia defined by EWGSOP $(60.9 \%$ vs $61.5 \%$, p-value = $0.96)$ were not statistically different between SA and nonSA patients. CRP level at the entrance to the hospital (47.44 vs 31.53 , p-value $=0.016)$, duration to get up and sit down 5 times $(21.15 \mathrm{~s}$ vs $12.73 \mathrm{~s}$, p-value $=0.05)$, were statistically different.

Conclusions: These preliminary data showed that older SA patients do not present more falls and sarcopenia. Inclusion is ongoing.

\section{FALLS AND SUBSEQUENT ADVERSE DRUG EVENTS AMONG ELDERLY-A REGISTER-BASED MATCHED CASE-CONTROL STUDY}

C. Rausch ${ }^{3,2}$, L. Laflamme ${ }^{3}$, S.E. de Rooij ${ }^{1}$, U. Bültmann ${ }^{2}$, J. Moller ${ }^{3}$, 1. University Medical Center Groningen, Department of Internal Medicine Groningen, University Center for Geriatric Medicine, Groningen, Netherlands,

2. University of Groningen, Unviersity Medical Center Groningen,Community and Occupational Medicine, Groningen, Netherlands, 3. Karolinska Institutet, Department of Public Health Sciences, Stockholm, Sweden

Epidemiological studies on the association between geriatric conditions like falls and subsequent adverse drug events (ADE) remain scarce among unselected community dwelling populations. This study investigates the sequential association between serious injurious falls and ADEs among older people, considering co-morbidity and prescribed medications.

A matched case-control study of Swedish residents 60 years and older was conducted. Cases with ADE were extracted from the National Patient and Death Registers from January 2006 to December 2009. Each case was matched with four controls by sex, age and residential area. Episodes of injurious falls were extracted from the NPR and information on dispensed medications during the four month period prior to index date from the Swedish Prescribed Drug Register. Effects were estimated with odds ratios (OR) and $95 \%$ confidence intervals (CI) using conditional logistic regression and adjusting for confounders, including comorbidity.

We found a three-fold increased risk of a new ADE in the six-month period after an injurious fall (OR 3.03; 95 $\%$ CI, $2.54-3.74)$. This increased risk was highest in the one to three week period after the fall injury, but remained high over the whole period. The risk was higher among the 60-79 year olds than $80+$ year olds. Those with an ADE one 